\title{
Daphnia magna tolerance to toxic cyanobacteria in the presence of an opportunistic infection within an evolutionary perspective
}

\author{
Alice Boudry, Sarah Devliegere, Shira Houwenhuyse, Lucas Clarysse, \\ Emilie Macke, Isabel Vanoverberghe \& Ellen Decaestecker
}

Aquatic Biology, IRF Life Sciences, Science \& Technology, KU Leuven, Campus Kortrijk, Belgium.

Corresponding author: ellen.decaestecker@kuleuven.be

\begin{abstract}
In aquatic environments, interactions between cyanobacteria and their grazers are crucial for ecosystem functioning. Cyanobacteria are photosynthetic prokaryotes, which are able to produce large blooms and associated toxins, some of which are able to suppress grazer fitness. Cyanobacterial blooms are intensified by global warming and eutrophication. In our experiments, the tolerance of Daphnia magna (Straus, 1820), an efficient grazer of toxic cyanobacteria, was studied. We used different $D$. magna clones sampled from different sediment depths, which corresponded to different time periods of eutrophication. Our results showed that different clones had a different tolerance towards the toxic cyanobacterial species, Microcystis aeruginosa, confirming the presence of genetic variation in D. magna tolerance to cyanobacteria. However, there was not a significant adaptive effect of sediment depth. As expected, in general under controlled, infection-free conditions M. aeruginosa reduced D. magna survival. However, a coincidental, non-intended opportunistic fungal infection in a first experiment allowed us to compare the response of D. magna to M. aeruginosa in infected individuals and non-infected individuals (from a second experiment). In the presence of this opportunistic infection, there was no negative effect of $M$. aeruginosa in the D. magna clones, suggesting that exposure to the infection provided protection for Daphnia individuals towards Microcystis. Biotic interactions can thus be important in the interpretation of cyanobacterial effects in zooplankton grazers and in finding appropriate solutions to reduce the occurrence of cyanobacterial blooms.
\end{abstract}

Keywords. Daphnia, toxic cyanobacteria, biotic interactions.

Boudry A., Devliegere S., Houwenhuyse S., Clarysse L., Macke E., Vanoverberghe I. \& Decaestecker E. (2020). Daphnia magna tolerance to toxic cyanobacteria in the presence of an opportunistic infection within an evolutionary perspective. Belgian Journal of Zoology 150: 81-93. https://doi.org/10.26496/2020.75

\section{Introduction}

Due to progressively common phenomena such as global warming, nutrient loading and eutrophication, cyanobacteria are becoming increasingly dominant in aquatic ecosystems (VISSER et al. 2016). These organisms can pose important threats to public health, as well as ecosystem structure and functioning, which is mainly due to their ability to produce toxic, secondary metabolites such as hepatotoxins and neurotoxins (De FigUEIREDO et al. 2004). Herbivorous zooplankton feed on these cyanobacteria and thus form an important link in the trophic chain, as they assure energy transfer from the primary producers 
to higher levels of the trophic chain (Kemal et al. 2014). Cyanobacteria cannot only limit herbivore fitness using toxins, they can also form colonies and filaments, which can cause clogging of the feeding apparatus of zooplankton species. Furthermore, cyanobacteria are deficient in sterols and unsaturated fatty acids, which are important elements in animal cell functioning. Consequently, cyanobacteria are of poor nutritional value for aquatic herbivores (KUSTER \& VON ELERT 2013).

The freshwater crustacean Daphnia magna was here used to study zooplankton - cyanobacteria interactions. Daphnia magna belongs to the Phylum Arthropoda and the Class Branchiopoda. Daphnia magna is a freshwater crustacean used as an ecological and evolutionary model organism, considering its high responsiveness to environmental stress, its key structuring role in freshwater ecosystems, its short life cycle and clonal reproduction. Because of its clonal reproduction, it is possible to study genotypic effects as possible factors influencing tolerance towards toxic cyanobacteria. Different responses of Daphnia genotypes to toxic cyanobacteria have been reported (Lemaire et al. 2012; MACKe et al. 2017a, 2020). Some Daphnia genotypes are able to control cyanobacterial blooms (PeretYatKo et al. 2012), while for others, fitness decreases upon exposure to cyanobacteria and a shift in zooplankton composition occurs (GHADOUANI et al. 2003). It has been suggested that these inconsistent results could be due to complex, genotype $\times$ genotype interactions (Lemaire et al. 2012). Different Daphnia genotypes may have different responses to the presence of cyanobacterial blooms and different cyanobacterial strains may have different defense mechanisms (e.g., different secondary toxic metabolites) against the zooplankton grazers. These genotype $\times$ genotype interactions play an important role in co-evolutionary processes. How Daphnia adapts to cyanobacteria is still debated. Throughout multiple generations, adaptation can occur through evolution, that is, by changing the Daphnia's genome or a shift in tolerant genotypes (HAIRSTON et al. 1999). Another possible explanation, however, is via the adaptation of the microbiome. The gut microbiota may play a crucial role in adaptation and acclimation of its host to rapid environmental change (ALBERDI et al. 2016), considering the fact that the microbiome composition and individual microbe genomes can change fast (MACKE et al. 2017b). A recent study conducted by MACKE et al. (2017a) found a strong impact of the microbiome on D. magna tolerance towards toxic cyanobacteria through gut microbiota transplant experiments. Another environmental biotic stress factor Daphnia shows adaptation to is parasitic infections. A high number of parasites from different taxa have been detected in Daphnia, with a variety of induced effects, including in the pond studied here (DeCAestecker et al. 2004; Pauwels et al. 2007). With respect to these parasites, strong genotype $\times$ genotype effects have been detected with coevolutionary interactions as a result (DECAESTECKER et al. 2007, 2013).

Another peculiar characteristic of Daphnia, which makes it an interesting model for studying tolerance evolution over time, is the fact that they can produce dormant eggs when conditions are not favorable. These eggs can remain in lake sediments for many years and hatch when conditions become favorable again (BRENDONCK \& De MeEsTer 2003). These resting eggs can be extracted from the sediments and revived in the laboratory by exposing them to the appropriate conditions. In this way, characteristics of individuals from different periods can be tested, such as tolerance towards cyanobacterial toxins. This method is called 'resurrection ecology' and provides researchers with the unique opportunity to study an evolutionary process in real time. In a study by HAIRSTON et al. (1999), researchers used this 'resurrection ecology' method in order to obtain individuals of Daphnia galeata (Sars, 1863) corresponding to different time periods and originating from different sediment layers of Lake Constance. This lake has been subjected to intense eutrophication, which caused a significant increase in cyanobacterial biomass. By hatching the eggs, it was found that the population of Daphnia became increasingly tolerant to a cyanobacteria containing diet.

The aim of this study was to measure and compare the tolerance of recent and older clones in a pond in Belgium (Oud-Heverlee), with changed environmental conditions over time (i.e., fish predation, 
BOUDRY A. et al., Daphnia tolerance to toxic cyanobacteria in the presence of an infection

eutrophication, ...). We exposed the different clones of D. magna to a random strain of Microcystis aeruginosa and tested for the evolution of tolerance of D. magna towards $M$. aeruginosa over time (Experiment 1). The clones of D. magna were derived from resting eggs in different sediment depths and thus corresponded to different time periods. Since cyanobacterial blooms have benefited from the increasing eutrophication and global warming through time (VISSER et al. 2016), our hypothesis was that recent clones of D. magna should have been associated with an increased exposure to toxic cyanobacteria. This hypothesis of increasing eutrophication is supported by the findings of REYSERHOVE et al. (2017) who found a temporal increase in nutrients in a pond "Abdij van 't Park" (OM2, Heverlee, Leuven) located $5.5 \mathrm{~km}$ from the Oud-Heverlee pond. For that reason, we hypothesized that D. magna from more recent sediment layers would be more tolerant to toxic cyanobacterial blooms. Due to an unexpected infection of an opportunistic fungus in Experiment 1 (in all experimental jars, in both nontoxic, control and toxic, experimental diets), we repeated this experiment in closed jars to exclude the effects of this infection. Nevertheless, the infection in Experiment 1 gave us the opportunity to explore the effects of a combined stressor on the fitness of Daphnia (i.e., fungus and toxic cyanobacteria).

\section{Material and methods}

The non-toxic green alga Chlorella vulgaris and the toxic cyanobacteria Microcystis aeruginosa strain (strain PCC 7806, toxic strain producing Microcystin-LR, provided by the Pasteur Culture Collection, Institut Pasteur, Paris, France) were both used as a food source for the D. magna individuals in our experiments. They were cultured in batch cultures under a light-dark cycle of $16: 8 \mathrm{~h}$ at a temperature of $20 \pm 2^{\circ} \mathrm{C}$ in $2 \mathrm{~L}$ jars, with constant stirring and aeration. For the cultivation of $M$. aeruginosa, direct exposure to light was avoided via screens around the culture jars. Wright's Cryptophyte (WC) medium (Guillard et al. 1972) was used for the cultivation of C. vulgaris, whereas WC without Tris medium was used for the cultivation of $M$. aeruginosa. The aeration system was provided with adapted filters $(22 \mu \mathrm{m})$ in order to avoid contamination. Ash-free dry weight of the algae was determined following MoHeimani et al. (2013): six filter papers ( 2 diets $\times 3$ replica) were placed in an oven at $100^{\circ} \mathrm{C}$ for 1 hour in order to remove any water on the filters. The filters were weighed and then used to filter $C$. vulgaris or M. aeruginosa. The filters were kept in the oven overnight, at $100^{\circ} \mathrm{C}$. They were then weighed and the carbon content was calculated using the values from the first and the second weighing.

Nine clones of D. magna were selected from a pond in Oud-Heverlee, Belgium. The studied pond is a manmade, 8.7 ha shallow pond that was used for fish culture (DECAESTECKER et al. 2013). The Daphnia hatched from resting eggs and were maintained in laboratory conditions for several years prior to the experiment. The resting eggs were isolated from three different sediment depths of a sediment core (3 clones/sediment depth). These three different sediment depths were characterized by different environmental conditions and the clones of $D$. magna have been described to have different life history strategies (Sтокs et al. 2016). The deepest section ranged from a depth of 18 to $21 \mathrm{~cm}$, corresponding to a period of 1970 to 1972. Sediment chronology is anchored in 1970 (the start of lacustrine sedimentation) and assumes conformable sediment deposition and a constant rate of sedimentation ( $\mathrm{g}$ dry sediment/ year) through time (CousYN et al. 2001). The clones from this bottom sediment depth were B7, B8 and B9. The middle section ranged from 11 to $14 \mathrm{~cm}$ and corresponded to the period of 1976 to 1979 . The clones that originated from the middle sediment depth were M5, M6 and M7. The top section, with a depth of $3 \mathrm{~cm}$, dated from 1988. The clones that were sampled from this sediment depth, were T2, T3 and T7. Three individuals were isolated per clone from the original culture and used to form independent maternal lines. These lines were grown in separate jars for two generations in order to reduce/eliminate possible maternal effects under saturating feeding conditions with C. vulgaris. Each maternal line was started with juveniles, maximum three days old of the second brood of the second generation. 
In Experiment 1, 20 individuals were isolated from each maternal line (Fig. 1). These individuals were then split into two diet treatment groups: a non-toxic $C$. vulgaris treatment group and a toxic $M$. aeruginosa treatment group. D. magna individuals were kept in open glass jars with filtered tap water $(80 \mathrm{~mL})$. In total there were 540 ( 9 clones $\times 2$ diets $\times 3$ replicates $\times 10$ individuals $)$ experimental units. The individuals of D. magna were fed with 1 milligram of carbon per liter every other day for the first eight days. The nontoxic diet treatment was given $100 \%$ C. vulgaris, whereas the toxic diet treatments were initially given $80 \%$ M. aeruginosa $-20 \%$ C. vulgaris. The percentage of $M$. aeruginosa from the toxic treatment was increased to $100 \%$ on day 15 of the experiment. During the experiment, all the specimens of D. magna were unexpectedly infected by an opportunistic parasite present in the lab (both non-toxic, control and toxic, experimental diets were affected), which was probably of fungal origin (Fig. 2, sequencing of the parasite is currently being performed). On day nine of the exposure, individuals of D. magna from both treatments did not show the expected somatic growth, which was probably due to the opportunistic infection. As a consequence, the carbon content fed to the individuals of D. magna was increased from one to two milligrams of carbon per liter, and secondly, all individuals of D. magna from both treatments were given an additional boosting treatment of $100 \mu \mathrm{l}$ of $100 \%$ C. vulgaris. Survival was monitored every other day during a period of 19 days. Juveniles were removed every other day.

In Experiment 2, we repeated Experiment 1, but with all individuals of D. magna kept individually in $80 \mathrm{~mL}$ (filtered tap water) closed Falcon tubes to avoid infection by the opportunistic fungus. This was performed to test our hypothesis that Daphnia from more recent sediment layers have a higher tolerance to toxic cyanobacteria than Daphnia from older/deeper sediment layers without the interference of the opportunistic infection. The experimental design is shown in Figure 1. A 100\% treatment with M. aeruginosa was used for the toxic treatment and a $100 \%$ treatment with C. vulgaris was used for the non-toxic treatment. The level of exposure of Microcystis was higher in Experiment 2 than in Experiment 1 in order to enhance the negative effect of the toxic cyanobacterial strain in the exposures.

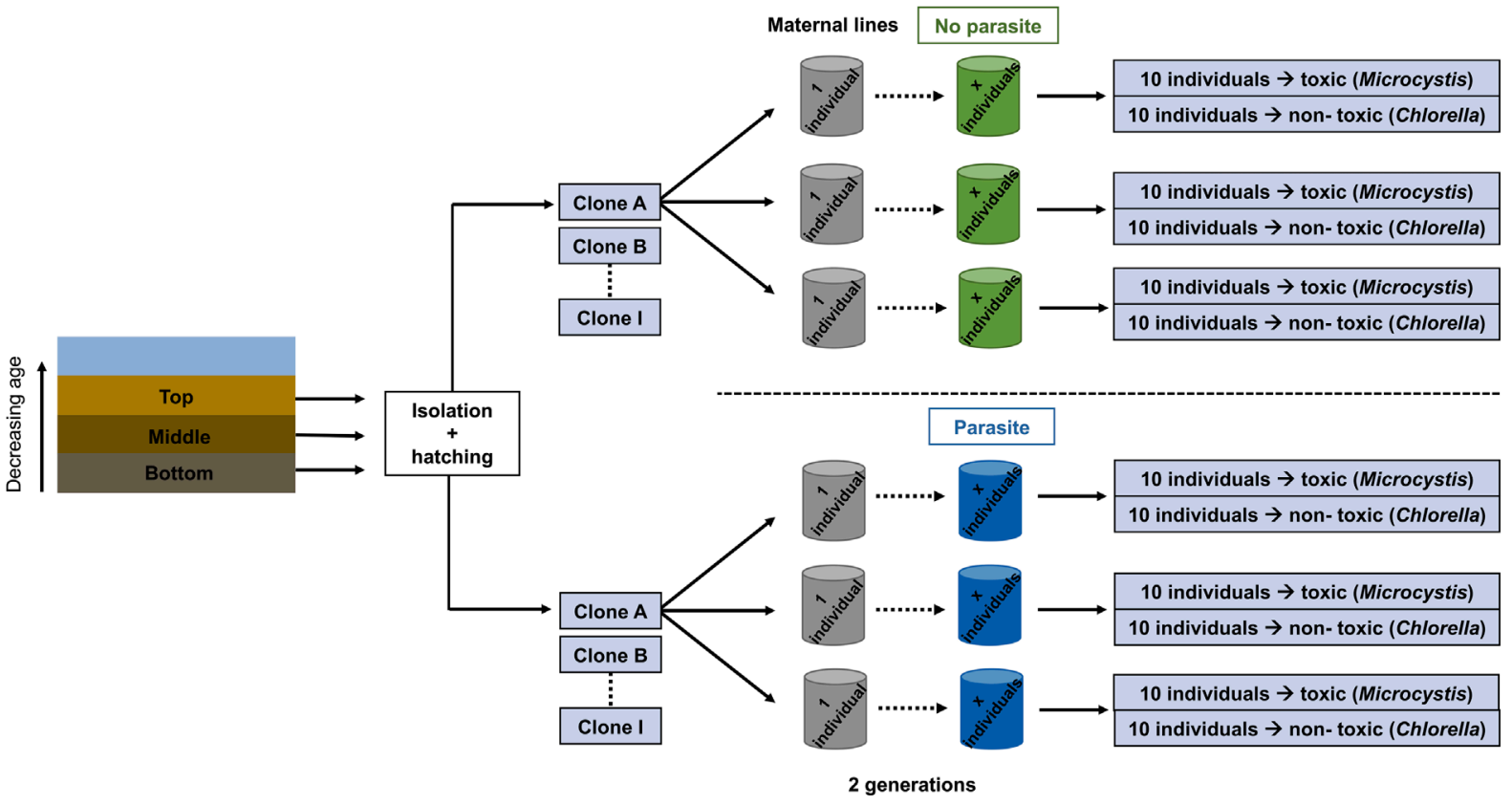

Fig. 1 - Visual representation of the two Experiments. In Experiment 1 a fungal infection affected the cultures of D. magna, in Experiment 2, there was no infection present. In both experiments the different clones of D. magna (9 in total, 3 from each subpopulation: Top, Middle and Bottom) were exposed to a $M$. aeruginosa (cyanobacterial) versus $C$. vulgaris (control) treatment. Per clone 10 individuals were raised in single experimental jars. 
Individuals of D. magna were fed every other day with 1.5 milligram of carbon per liter (intermediate food level of the feeding conditions between the two weeks of the experiment). This experiment was performed for one week, as the mortality rate in the M. aeruginosa treatment was very high. In this Experiment 2, we did not observe any parasitic infection. Additionally, individuals of D. magna that were still alive after one week were isolated and measured using a stereo microscope. The length of an individual of D. magna was determined as the distance between the top of its head and the base of its apical spine. Sizes of corresponding individuals of D. magna from the two treatments were compared, which allowed us to investigate the effect of M. aeruginosa on individual somatic growth.

A survival analysis using a Cox Proportional Hazards model (PHREG procedure in SAS 9.4) was conducted on the data to estimate the effect of the following factors on survival: 'Parasite' (equal as 'Experiment'), 'Sediment Depth', 'Clone' and 'Diet', as well as their interactions. Furthermore, the dataset contained a 'censored data' variable as an upper censor was placed on survival. Hereby, all specimens of $D$. magna that survived longer than seven days were determined in the dataset as individuals of $D$. magna with a survival time of seven days. This way, it was possible to compare the results of Experiment 2, which lasted 7 days, and Experiment 1, which lasted longer. The analysis was performed using SAS 9.4. A one-tailed t-test was performed in order to compare the sizes of individuals of D. magna from the treatments with C. vulgaris and M. aeruginosa in Experiment 2.

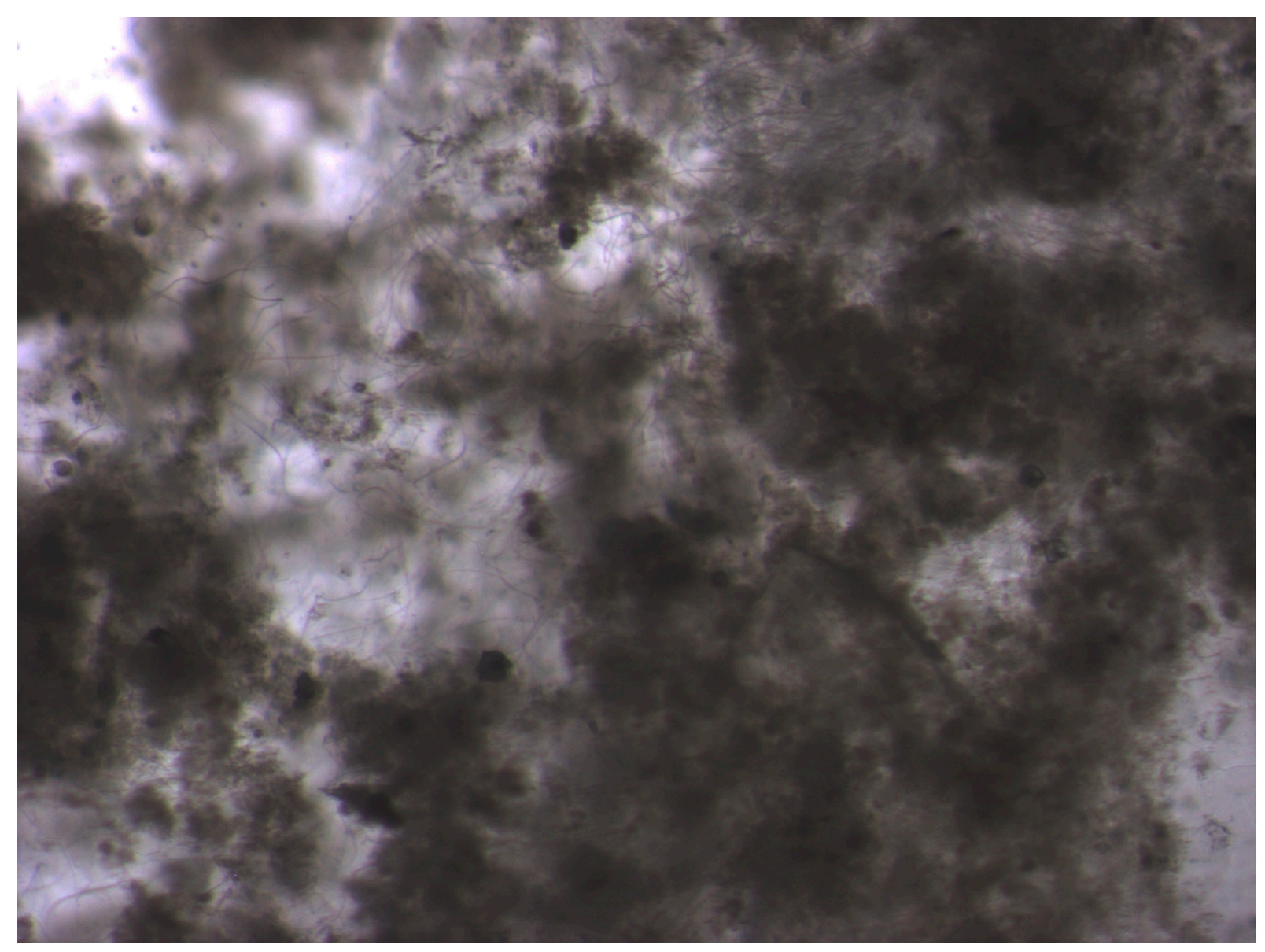

Fig. 2 - Parasite sampled from the water of individuals of D. magna in Experiment 2 (Olympus stereo microscope, $100 \times$ magnification). Characterization of the fungus is currently being carried out by sequencing. This opportunistic infection causes severe mortality to the Daphnia lab cultures and is recurrent in different labs throughout Europe. 


\section{Results}

\section{Results of Experiment 1}

In the presence of the opportunistic infection (Fig. 2 most likely a fungal infection, given the typical hyphae present), the survival analysis showed no significant interactions or main effects (Fig. 3). Results of the Cox proportional hazards model analysis inclusive Sediment Depth: Diet: $p=0.8546$, Sediment Depth: $\mathrm{p}=0.1379$, Diet*Sediment Depth: $\mathrm{p}=0.7046$, Clone (Sediment Depth): $\mathrm{p}=0.3131$, Diet*Clone (Sediment Depth): $\mathrm{p}=0.1379$. A second analysis was carried out in which the factor sediment depth was excluded and results are shown in Table 1: there was no significant Diet*Clone interaction or main Diet or Clone effect.

TABLE 1

Results of Cox Analysis on D. magna Survival in Experiment 1 (Type 3 test).

\begin{tabular}{lccccc}
\hline Effect & Khi-2 Wald & DDL & P $>$ Khi-2 & DDL adjusted & P $>$ Khi-2 adjusted \\
\hline Diet & 0.03 & 1 & 0.85 & 0.99 & 0.85 \\
Clone & 4.26 & 8 & 0.83 & 2.62 & 0.19 \\
Diet*Clone & 3.85 & 8 & 0.87 & 7.99 & 0.87 \\
Id & 52.93 & & & 10.78 & $<0.0001$ \\
\hline
\end{tabular}

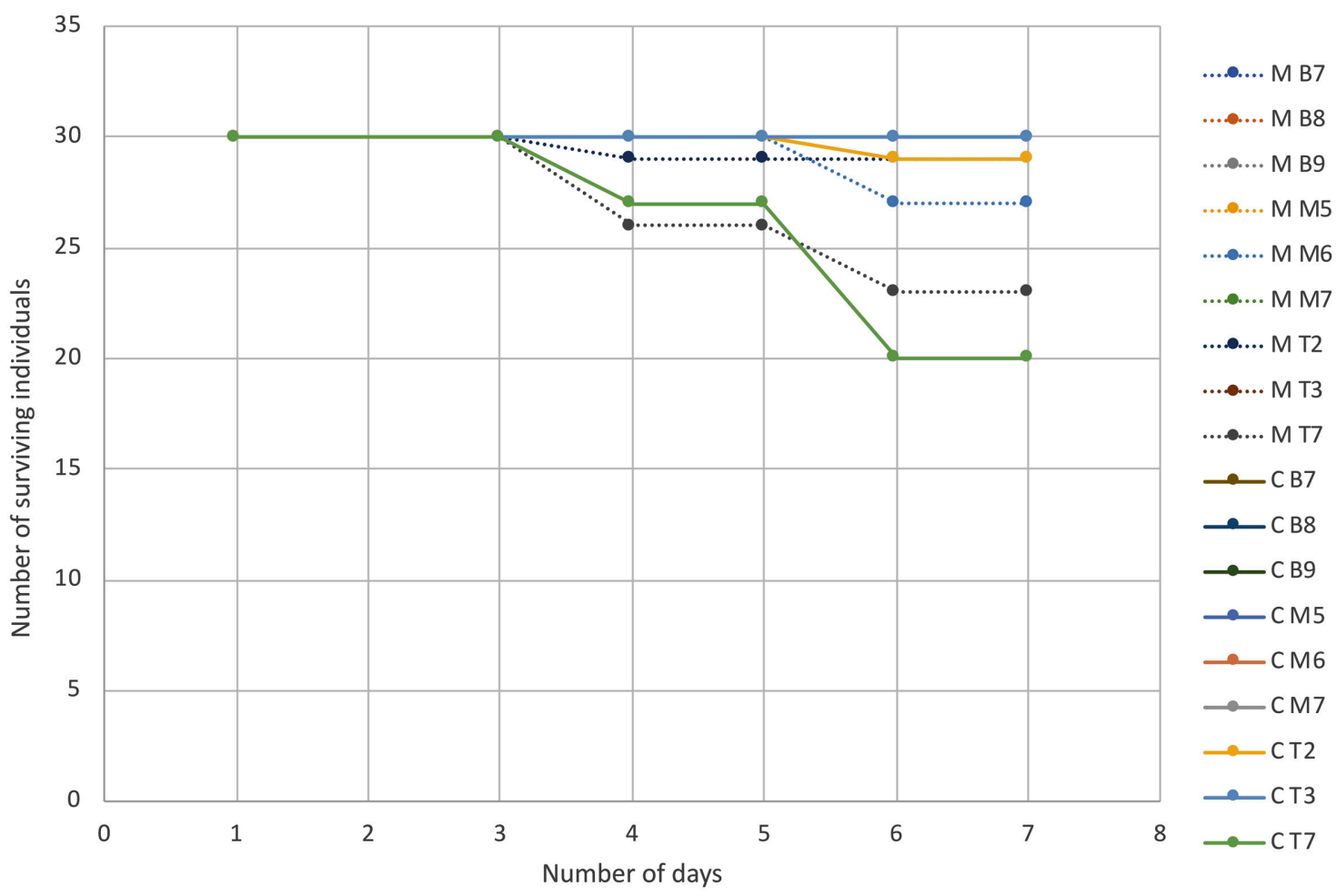

Fig. 3 - Survival of D. magna in Experiment 1 (in the presence of the fungal infection). Colors indicate the different genotypes and line type indicates the different diets (dotted: M. aeruginosa; full: C. vulgaris). 


\section{Results of Experiment 2}

There was no significant 'Sediment Depth' effect on the survival of Daphnia, so this factor was taken out of the Cox proportional hazards model. In the absence of the parasite, there was a marginally significant effect of the 'Diet*Clone' interaction (Table 2, $\mathrm{p}=0.057$ ). The D. magna clones T2, B9, M6, B8 and M7 had a lower survival rate for the treatment with Microcystis than the other clones (Fig. 4). A significant main effect for 'Diet' (Table 2, $\mathrm{p}<0.0001$ ) was present: individuals of $D$. magna had a lower survival in the treatment with M. aeruginosa than in that with C. vulgaris (Fig. 4). Individuals of D. magna from the treatment with $M$. aeruginosa were also significantly smaller than those D. magna from the treatment with Chlorella (One tailed t-test; $\mathrm{p}=1,52163 \mathrm{E}-14$, see Fig. 5).

TABLE 2

Results of Cox Analysis on D. magna Survival in Experiment 2 (Type 3 test).

\begin{tabular}{cccccc}
\hline Effect & Khi-2 Wald & DDL & P $>$ Khi-2 & DDL adjusted & P $>$ Khi-2 adjusted \\
\hline Diet & 170.2 & 1 & $<0.0001$ & 0.99 & $<0.0001$ \\
Clone & 6.6 & 8 & 0.574 & 4.38 & 0.188 \\
Diet*Clone & 15.1 & 8 & 0.057 & 7.97 & 0.057 \\
Id & 34.03 & & & 12.1 & 0.0007 \\
\hline
\end{tabular}

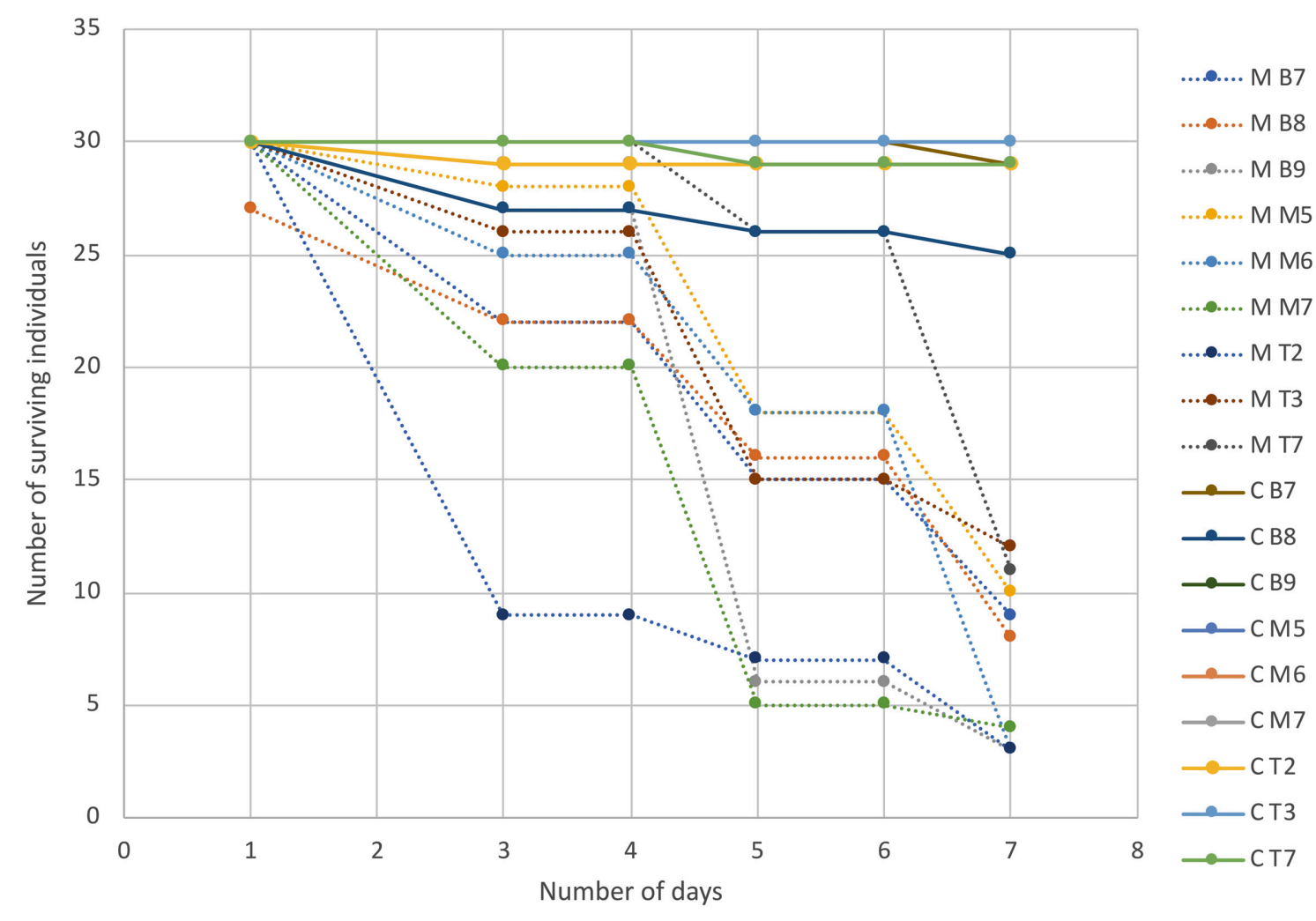

Fig. 4 - Survival of D. magna in Experiment 2 (in the absence of the fungal infection). Colors indicate the different genotypes and line type indicates the different diets (dotted: M. aeruginosa; full: C. vulgaris). 


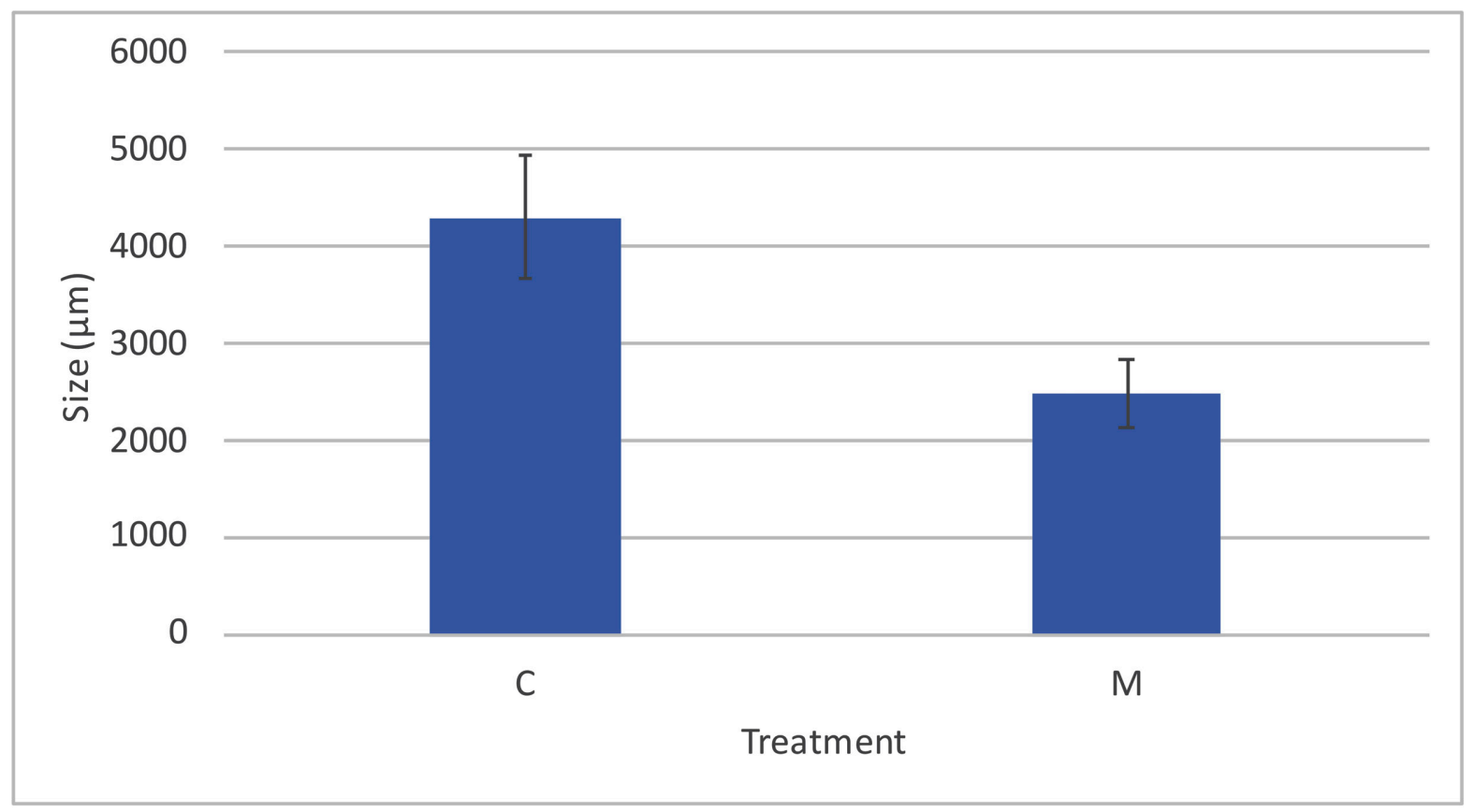

Fig. 5 - Daphnia magna body size in the C. vulgaris treatment (C, Control) vs the $M$. aeruginosa treatment (M). The error bars represent the standard deviation of the D. magna body size in the C. vulgaris or the M. aeruginosa treatment.

\section{Survival Analysis, combined over both experiments}

A survival analysis using the Cox proportional hazards model was performed on the combined data from the two experiments (i.e., in both the absence and the presence of the fungal parasite, so 'parasite' effect is equal to 'experiment' effect). There was no significant sediment depth effect or interaction with sediment depth, thus the survival analysis was performed without sediment depth as a factor. There was a 'Parasite*Diet' interaction (Table 3, p < 0.0001) and the factors 'Parasite' (Table 3, p < 0.0001) and 'Diet' (Table 3, $\mathrm{p}<0.0001$ ) were significant. This was due to the higher survival rate for individuals of D. magna from Experiment 1 (in the presence of the parasite) than for those from Experiment 2 (in the absence of the parasite, Fig. 6, 'Parasite' or 'Experiment' effect). In Experiment 1 (in the presence of the parasite), the significant diet effect detected in Experiment 2 (in the absence of the parasite) was lost ('Parasite*Diet' interaction, Fig. 6). The 'Parasite *Clone' interaction $(\mathrm{p}=0.0565)$ was marginally significant. Over both experiments, there was no significant Clone*Diet effect (Table 3, $\mathrm{p}=0.4510$ ).

\section{Discussion}

Two experiments were performed to investigate whether Daphnia from more recent years have a higher tolerance against toxic cyanobacteria than Daphnia from older years. In the first experiment open glass jars were used. During this first experiment Daphnia became infected by an opportunistic infection present in the lab. Because of this, the experiment was repeated with Daphnia in closed falcon tubes in Experiment 2.

The second experiment showed a decreased survival of D. magna when fed with toxic M. aeruginosa compared to the non-toxic control $C$. vulgaris diet. The body size of D. magna was also significantly smaller in the diet with $M$. aeruginosa than in the control diet in this experiment. This lower survival and reduced body size upon exposure to M. aeruginosa can be explained by cyanobacterial toxicity and 
TABLE 3

Results of Cox Analysis on D. magna Survival in Experiment 1 and 2 (Type 3 test).

\begin{tabular}{lccccc}
\hline Effect & Khi-2 Wald & DDL & P $>$ Khi-2 & DDL adjusted & P $>$ Khi-2 adjusted \\
\hline Parasite & 31.66 & 1 & $<0.0001$ & 0.461 & $<0.0001$ \\
Diet & 60.29 & 1 & $<0.0001$ & 0.998 & $<0.0001$ \\
Parasite*Diet & 54.43 & 1 & $<0.0001$ & 0.998 & $<0.0001$ \\
Clone & 4.88 & 8 & 0.769 & 3.581 & 0.2473 \\
Parasite*Clone & 8.52 & 8 & 0.384 & 3.585 & 0.0565 \\
Diet*Clone & 7.82 & 8 & 0.451 & 7.994 & 0.451 \\
Parasite*Diet*Clone & 3.15 & 8 & 0.925 & 7.994 & 0.9245 \\
Id & 95.1992 & & & 23.23 & $<0.0001$ \\
\hline
\end{tabular}

their low nutritional value. In addition, feeding inhibition of all algal species exposed (i.e., stop feeding as defensive response) by specimens of $D$. magna may have played a role as well (LürLING et al. 2003). The strength of this negative effect of $M$. aeruginosa varied among the clones of D. magna (marginal significant Diet*Clone interaction), confirming the presence of genetic variation in the tolerance of D. magna to cyanobacteria, as observed in previous studies (MACKE et al. 2017a; LemAIRE et al. 2012). The clones T2, B9, M6, B8 and M7 had a strong decrease in survival, whereas the clones T3, T7, B7, B8 and M5 were less affected by the presence of M. aeruginosa (Fig. 4).

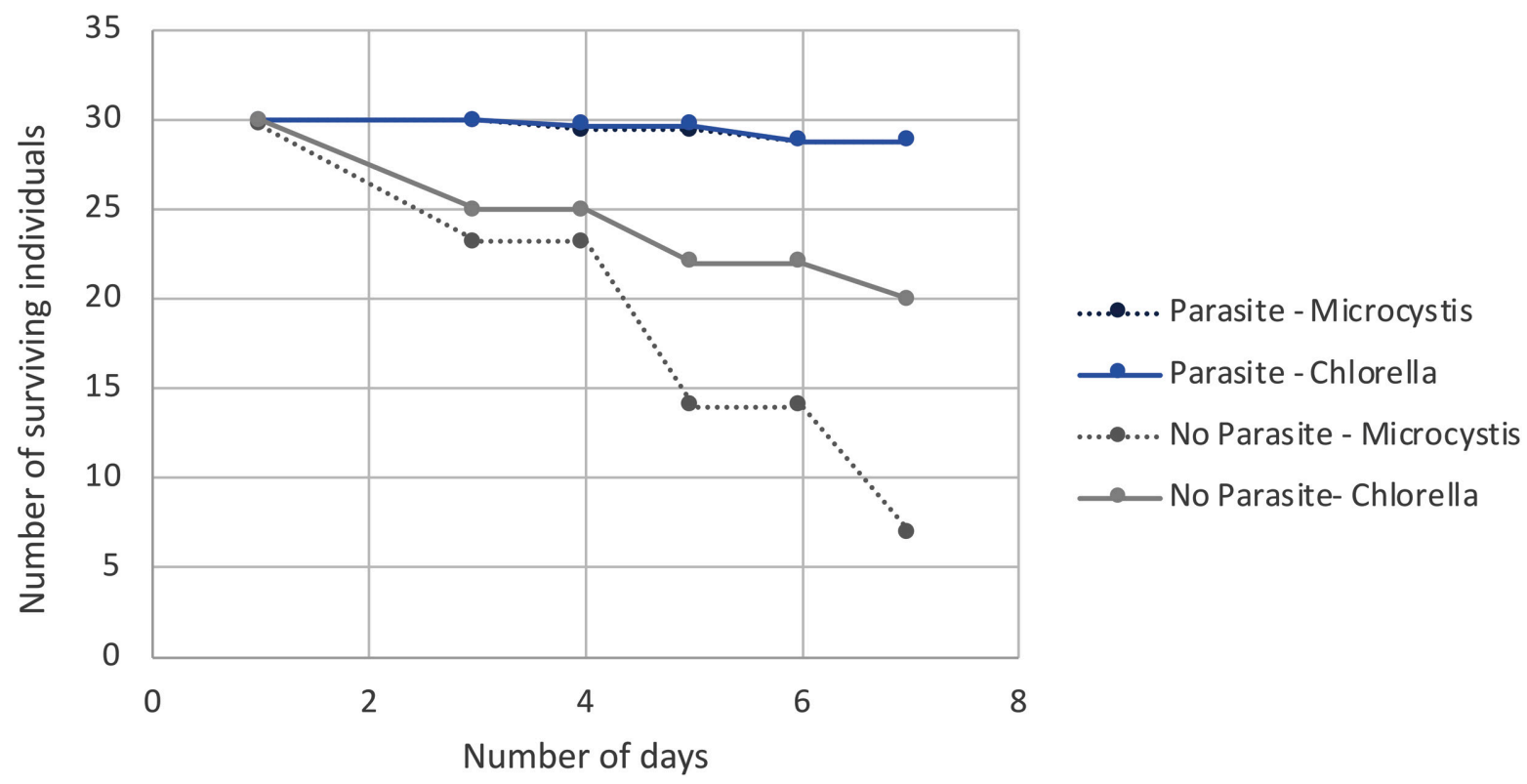

Fig. 6 - Survival of D. magna in Experiment 1 versus Experiment 2 in different diet treatments. Colors indicate the different experiments (Blue: Experiment 1; Grey: Experiment 2) and line type indicates the different diets (dotted: M. aeruginosa; full: C. vulgaris). 
No significant 'Sediment Depth*Diet' interaction was present, in none of the experiments, which shows that the response of D. magna to M. aeruginosa was not affected by the sediment depth from which the different clones of D. magna originated (as was determined in HaIRSTON et al. 1999). This result contradicts the hypothesis that individuals of D. magna from more recent pond sediment depths would be more tolerant towards $M$. aeruginosa, than individuals originating from older sediment depths, as they would have had the time to adapt to increasingly eutrophic conditions. A possible explanation for these results is that in our experiments a random strain of Microcystis was used, Microcystis aeruginosa PCC7806. The Daphnia used in this experiment had never been exposed to this toxic strain of Microcystis before. LEMAIRE et al. (2012) showed that genotype $\times$ genotype interactions were important in determining the tolerance of genotypes of Daphnia to those of Microcystis. The increased tolerance to toxic cyanobacteria in the more recent genotypes of Daphnia observed in HAIRSTON et al. (1999) may have occurred because the researchers used a strain of Microcystis that was present in the pond from which the Daphnia originated. In addition, HaIRston et al. (1999) monitored the growth rate, while we only looked at survival and body size at the end of the experiment in the second experiment (in absence of the parasite).

In contrast to the second experiment (in absence of the infection), we did not observe a negative effect of $M$. aeruginosa in the first experiment (in the presence of the parasite) (see combined analysis over both experiments: 'Parasite*Diet' interaction). In the presence of the opportunistic infection, there was no difference in survival upon exposure to $M$. aeruginosa compared to the exposure to $C$. vulgaris as in Experiment 1. This effect may be explained through a protective effect of the infection on D. magna towards $M$. aeruginosa. Such a protective effect could be linked to a parasite-mediated reduction in toxicity of M. aeruginosa. This conclusion corresponds with findings of MOHAMED et al. (2014) and JIA et al. (2012), who demonstrated the biodegradation of M. aeruginosa by the algicidal fungus Trichoderma citrinoviride and the biodegradation of Microcystin-LR by Trichaptum abietum, respectively. The removal or lysation of algal cells by fungi can happen indirectly through the production of extracellular substances or by degrading them directly after encasing the algae in a mucous membrane. This biodegradation is frequently accompanied with detoxification. The reduced toxicity of the cyanobacteria may offset the feeding inhibition in D. magna and, although this effect would not affect the low nutritional value of $M$. aeruginosa, it could explain the higher survival of D. magna upon cyanobacterial exposure in Experiment 1. Moreover, recent research showed that chytrid fungi may even provide a food source for zooplankton through the production of zoospores and that chytrid infections make cyanobacteria a more valuable food source, which may offset feeding inhibition and low nutritional values and may ultimately contribute to the protective effect suggested by our study (FrENKEN et al. 2018). Further examples of similar biotic interactions are found in the literature. A study conducted by CoOPMAN et al. (2014) suggested a protective effect of M. aeruginosa on D. magna against White Fat Cell Disease, which is a viral infection (CoOpman et al. 2014; ToEnSHOFF et al. 2018). Recently, SANCHEZ et al. (2019) detected a medicinal effect of cyanobacteria on Daphnia dentifera increasing its tolerance to parasitism. These results suggest that biotic interactions may be important in the response of zooplankton towards particular stressors, given that these biotic interactions can interact with and even weaken the toxic effects of an antagonist. Alternatively, it can be that the host is boosted by the presence of one stressor and that defenses towards other antagonists (with particular toxins) is increased. Intriguingly, in this same population of Daphnia, we earlier detected an increase in fungal disease tolerance, in terms of increased Heat Shock production, through time (PAuwels et al. 2007). Nevertheless, these responses have been determined as being specific, so it is unlikely that there is a general induced response towards different types of biotic antagonists (herbivore and parasite). This specificity is confirmed in Experiment 1 through the interaction effect between clones of D. magna and the fungal parasite showing that the clones responded in a specific way to the fungal infection, as is generally detected in Daphnia-parasite interactions (DECAESTECKER et al. 2007). 
BOUDRY A. et al., Daphnia tolerance to toxic cyanobacteria in the presence of an infection

It is important to note that the difference between the two experiments may have been an effect of the two experiments (absence/presence parasite) not being carried out at the same time. Although the same cyanobacterial strain was tested, it may be that subtle differences in toxicity may have been present in both experiments. The percentage of $M$. aeruginosa, used in Experiment 1 (presence of parasite) was also slightly lower (80\%) than the percentage used in Experiment 2 (absence of parasite, 100\%). This could partly explain the higher survival rates in Experiment 1 . However, a recent study by MACKE et al. (2017a) exposed the same B7 clone (= "S2") to an 80\% treatment of the same cyanobacterial strain (PCC 7806), which caused an approximate 55\% reduction in survival after seven days. Thus, even an $80 \%$ treatment with $M$. aeruginosa has strong negative effects on the survival of $D$. magna (MACKE et al. 2017a) and it is thus very unlikely that the higher survival rates in Experiment 1 are completely due to the difference in percentages of M. aeruginosa.

In conclusion, toxic cyanobacterial exposure significantly affected the survival and growth of D. magna. The exposure of $M$. aeruginosa induced a lower survival of D. magna, as well as decreased somatic growth with a trend for genetic specificity of the clones of D. magna in their cyanobacterial tolerance. However, in the presence of a fungal infection the negative effect of the toxic strain of Microcystis disappeared. The results of the present experiment indicate that certain types of parasitic infection could have a protective effect on individuals of D. magna towards cyanobacterial toxins. It is important to perform comprehensive follow-up experiments to confirm the present results and to provide further knowledge on these biotic interactions, as they could be important in finding appropriate solutions to reduce the occurrence of cyanobacterial blooms.

\section{Acknowledgements}

Author Contributions: Conceptualization, A.B., S.D., L.C., E.M. and E.D.C.; Methodology, I.O. E.M., A.B., S.D., L.C.; Formal analysis, A.B., E.M.; writing-original draft preparation, A.B., S.D., L.C., E.M., E.D.C.; writing-review and editing, all authors; funding acquisition, E.D.C.

Funding: Funding has been provided to EDC via FWO G.0643.13, FWO G.092619N and the KU Leuven research funding C16/17/002.

\section{References}

Alberdi A., Aizpurua O., Bohmann K., Zepeda-Mendoza M.L. \& Gilbert M.T.P. (2016). Do vertebrate gut metagenomes confer rapid ecological adaptation? Trends in Ecology and Evolution 31: 689-699. https://doi.org/10.1016/j.tree.2016.06.008

Brendonck L. \& De Meester L. (2003). Egg banks in freshwater zooplankton: evolutionary and ecological archives in the sediment. Hydrobiologia 491: 65. https://doi.org/10.1023/A:1024454905119

Coopman M., Muylaert K., Lange B., Reyserhove L. \& Decaestecker E. (2014). Context dependency of infectious disease: the cyanobacterium Microcystis aeruginosa decreases White Fat Cell Disease in Daphnia magna. Freshwater Biology 59: 714-723. https://doi.org/10.1111/fwb.12298

Cousyn C., De Meester L., Colbourne J.K., Brendonck L., Verschuren D. \& Volckaert F. (2001). Rapid, local adaptation of zooplankton behavior to changes in predation pressure in the absence of neutral genetic changes. Proceeding of the National Academy of Sciences 98: 6256-6260. https://doi.org/10.1073/pnas.111606798

Decaestecker E., Levever C., De Meester L., Ebert E. (2004). Haunted by the past: evidence for dormant stage banks of microparasites and epibionts of Daphnia. Limnology and Oceanography 49: 1355-1364. https://doi.org/10.4319/1o.2004.49.4_part_2.1355 
Decaestecker E., Gaba S., Raeymaekers J.A.M., Stoks R., Van Kerckhoven L., Ebert D. \& De MEESTER L. (2007). Host-parasite 'Red Queen' dynamics archived in pond sediment. Nature 450: 870874. https://doi.org/10.1038/nature06291

Decaestecker E., De Gersem H., Michalakis Y. \& Raeymaekers J. (2013). Damped long-term hostparasite Red Queen coevolutionary dynamics: a reflection of dilution effects? Ecology Letters 16: 14551462. https://doi.org/10.1111/ele.12186

De Figueiredo D.R., Azeiteiro U.M., Esteves S.M., Gonçalves F.J.M. \& Pereira M.J. (2004). Microcystin-producing blooms - A serious global public health issue. Ecotoxicology and Environmental Safety 59: 151-163. https://doi.org/10.1016/j.ecoenv.2004.04.006

Frenken T., Wierenga J., van Donk E., Declerck S.A.J., De Senerpont Domis L.N., Rohrlack T. \& VAN DE WAAL D.B. (2018). Fungal parasites of a toxic inedible cyanobacterium provide food to zooplankton. Limnology and Oceanography 63 (6): 2384-2393. https://doi.org/10.1002//no.10945

Ghadouani A., Pinel-Alloul B. \& Prepas E.E. (2003). Effects of experimentally induced cyanobacterial blooms on crustacean zooplankton communities. Freshwater Biology 48: 363-381. https://doi.org/10.1046/j.1365-2427.2003.01010.x

Guillard R.R. \& LoREnzen C.J. (1972). Yellow-green algae with chlorophyllide C1, 2. Journal of Phycology 8: 10-14. https://doi.org/10.1111/j.1529-8817.1972.tb03995.x

Hairston N.G., Lampert W., Caceres C.E., Holtmeier C.L., Weider L.J., Gaedke U., Fischer J.M., Fox J.A. \& Post D.M. (1999). Lake ecosystems: Rapid evolution revealed by dormant eggs. Nature 401: 446. https://doi.org/10.1038/46731

JiA Y., Du J., Song F., Zhao G. \& Tian X. (2012). A fungus capable of degrading Microcystin-LR in the algal culture of Microcystis aeruginosa PCC7806. Applied Biochemistry and Biotechnology 166: 987-996. https://doi.org/10.1007/s12010-011-9486-6

Kemal A.G., Hansson L.A. \& LÜRLING M. (2014). Understanding cyanobacteria-zooplankton interactions in a more eutrophic world. Freshwater Biology 59: 1783-1798.

https://doi.org/10.1111/fwb.12393

Kuster C.J. \& Von Elert E. (2013). Interspecific differences between D. pulex and D. magna in tolerance to cyanobacteria with protease inhibitors. PloS one 8: 1-8.

https://doi.org/10.1371/journal.pone.0062658

Lemaire V., Brusciotti S., Van Gremberghe I., Vyverman W. \& Vanoverbeke J. (2012). Genotype × genotype interactions between the toxic cyanobacterium Microcystis and its grazer, the waterflea. Evolutionary Applications 5: 168-182. https://doi.org/10.1111/j.1752-4571.2011.00225.x

LÜRLING M. (2003). Daphnia growth on microcystin-producing and microcystin-free Microcystis aeruginosa in different mixtures with the green alga Scenedesmus obliquus. Limnology and Oceanography 48: 2214-2220. https://doi.org/10.4319/1o.2003.48.6.2214

Macke E., Callens M., De Meester L. \& Decaestecker E. (2017a). Host genotype-dependent gut microbiota drives zooplankton tolerance to toxic cyanobacteria. Nature Communications 8: 1608. https://doi.org/10.1038/s41467-017-01714-x

Macke E., Tasiemski A., Massol F., Callens M. \& Decaestecker E. (2017b). Life history and ecoevolutionary dynamics in light of the gut microbiota. Oikos 126: 508-538.

https://doi.org/10.1111/oik.03900

Macke E., Callens M., Massol F., Vanoverberghe I., De Meester L. \& Decaestecker E. (2020). Diet and genotype of an aquatic invertebrate affect the composition of free-living microbial communities. Frontiers in Microbiology in press. https://doi.org/10.3389/fmicb.2020.00380 
Mohamed Z.A., Hashem M. \& Alamri S.A. (2014). Growth inhibition of the cyanobacterium Microcystis aeruginosa and degradation of its microcystin toxin by the fungus Trichoderma citrinoviride. Toxicon 86: 51-58. https://doi.org/10.1016/j.toxicon.2014.05.008

Moheimani N.R., Borowitzka M.A., Isdepsky A. \& Sing S.F. (2013). Standard Methods for Measuring Growth of Algae. In: Borowitzka M.A. \& Moheimani N.R. (eds) Algae for Biofuels and Energy: 265-284. Springer, Dordrecht. https://doi.org/10.1007/978-94-007-5479-9

Pauwels K., Stok R., Decaestecker E. \& De Meester L. (2007) Evolution of Heat Shock protein expression in a natural population of Daphnia magna. American Naturalist 170: 800-805. https://doi.org/10.1086/521956

Peretyatko A., Teissier S., De Backer S. \& Triest L. (2012). Biomanipulation of hypereutrophic ponds: when it works and why it fails. Environmental Monitoring and Assessment 184: 1517-1531. https://doi.org/10.1007/s10661-011-2057-z

Reyershove L., Samaey G., Muylaert K., Coppé V., Van Colen W. \& Decaestecker E. (2017). A historical perspective of nutrient change impact on an infectious disease in Daphnia. Ecology 98 (11): 2784-2798. https://doi.org/10.1002/ecy.1994

SÁnchez K.F., Huntley N., Duffy M.A. \& HunTer M.D. (2019). Toxins or medicines? Phytoplankton diets mediate host and parasite fitness in a freshwater system. Proceedings of the Royal Society B 286: 1894. https://doi.org/10.1098/rspb.2018.2231

Stoks R., Govaert L., Pauwels K., Jansen B. \& De Meester L. (2016). Resurrecting complexity: the interplay of plasticity and rapid evolution in the multiple trait response to changes in predation pressure in the water flea Daphnia magna. Ecology Letters 19: 180-190. https://doi.org/10.1111/ele.12551

Toenshoff E.R., Fields P.D., Bourgeois Y.X. \& Ebert D. (2018). The end of a 60-year riddle: Identification and genomic characterization of an Iridovirus, the causative agent of White Fat Cell Disease in zooplankton. G3: Genes, Genomes, Genetics 8 (4): 1259-1272.

https://doi.org/10.1534/g3.117.300429

Visser P.M., Verspagen J.M.H., Sandrini G., Stal L.J., Matthijs H.C.P., Davis T.W., Pearl H.W. \& HuisMAN J. (2016). How rising CO2 and global warming may stimulate harmful cyanobacterial blooms. Harmful Algae 54: 145-159. https://doi.org/10.1016/j.hal.2015.12.006

Manuscript received: 22 June 2019

Manuscript accepted: 15 June 2020

Published on: 7 July 2020

Branch editor: Merlijn Jocqué 